\title{
Parietal Cortical Neuronal Activity Is Selective for Express Saccades
}

\author{
Mo Chen (陈默, ${ }^{1 *}$ Yu Liu (刘㫜, ${ }^{1 \star}$ Linyu Wei (魏林有), 2 and Mingsha Zhang (张吗沙) ${ }^{1}$ \\ ${ }^{1}$ Institute of Neuroscience, State Key Laboratory for Neuroscience, Shanghai Institutes for Biological Sciences, Chinese Academy of Sciences, Shanghai, \\ China 200031, and 2Department of Physiology and Neurobiology, Xinxiang Medical College, Xinxiang, China 453003
}

Saccadic eye movements are central to primate behavior and serve to move the eyes to visual objects of interest. Express saccades, unlike regular saccades, occur with very short reaction times, a behavior necessary for speeded reactions in goal-directed behavior. Previous studies have shown that introduction of a blank interval (gap) between the fixation point offset and the saccadic target onset leads to an increase in the number of express saccades and that the superior colliculus plays a crucial role in the generation of express saccades. A longstanding hypothesis asserted that express saccades are mediated largely by a subcortical circuit, circumventing extrastriate visual cortex. An alternative "posterior pathway" hypothesis proposed the involvement of posterior parietal cortex. In the present study, using a gap saccade task, we investigated the role of nonhuman primate's lateral intraparietal cortex (LIP) in generation of express saccades. We show that roughly half of recorded LIP neurons were modulated during the gap interval. Moreover, a group of neurons with persistent activity in a memory-guided saccade task enhanced their activity during express saccades relative to that during regular saccades. After reducing the target's certainty by increasing the potential target locations, neuronal activity remained in the similar level during express saccades but markedly reduced during regular saccades that correlated with the increase of saccadic reaction time in the regular saccade. Our results suggest that area LIP is directly involved in generating saccades in express mode.

\section{Introduction}

Saccadic eye movements, which shift the eyes to objects of interest, are of great importance to primate behavior. Some saccades occur with very short reaction time and, moreover, occur frequently when the eyes move to a previously predicted location. Such express saccades are advantageous for speeded reactions in goal-directed behavior (Sokolov, 1963). To study express saccades, a saccade task called the gap task was designed to increase the incidence of express saccades. In gap tasks, rather than target onset occurring simultaneously with fixation spot offset, a delay (gap) precedes target onset (Fischer and Boch, 1983; Fischer and Ramsperger, 1984). This gap interval is believed to permit preparatory activity that then leads to a speeded saccade.

Single neuron recordings from monkey superior colliculus (SC) performing the gap saccade task exhibited greater activity during express saccades than during regular saccades (Edelman and Keller, 1996; Dorris et al., 1997; Sparks et al., 2000; Boehnke and Munoz, 2008), but such express saccade enhanced activity was not found in frontal eye field (FEF) (Dias and Bruce, 1994;

\footnotetext{
Received June 4, 2012; revised 0ct. 6, 2012; accepted Nov. 12, 2012.

Author contributions: Y.L. and M.Z. designed research; M.C., Y.L., and L.W. performed research; M.C. and Y.L. analyzed data; M.Z. wrote the paper.

This study was supported by the following foundations: The Hundred Talent Program, Chinese Academy of Sciences; 973 program (2100CBA00400), Chinese government; Pujiang Program, Shanghai government; State Key Laboratory of Neuroscience, Chinese government.

The authors declare no competing financial interests.

*M.C. and Y.L. contributed equally to this work.

Correspondence should be addressed to Mingsha Zhang, 320 Yueyang Road, Building 23, Room 305, Shanghai, People's Republic of China 200031. E-mail: mingsha@ion.ac.cn.

DOI:10.1523/JNEUROSCI.2675-12.2013

Copyright $\odot 2013$ the authors $\quad 0270-6474 / 13 / 330814-10 \$ 15.00 / 0$
}

Everling and Munoz, 2000). An influential hypothesis proposed that express saccades are mediated by a Retina-V1-SC circuit (Isa, 2002). Notably, this hypothesis excluded the involvement of the higher cortical regions in express saccade generation. In contrast, another line of experiments observed that, following lesions of posterior parietal cortex in both human and nonhuman primates, express saccades were rare and saccade reaction times (SRTs) increased (Lynch and McLaren, 1989; Braun et al., 1992; Li et al., 1999). Consistent with this, EEG studies in humans found increased activity in an occipital-parietal network during the gap interval preceding express saccades (Hamm et al., 2010), further suggesting that parietal areas might be specifically involved in generation of express saccades. Such findings support an alternative posterior pathway proposal that strongly implicates posterior parietal cortex (PPC) in express saccade generation (Schiller and Tehovnik, 2001).

To examine at a neural level the role of PPC in express saccade behavior, we have recorded from neurons in the lateral inferior parietal cortex (LIP) in the macaque monkey, an area of PPC known to be heavily involved in eye saccade behavior. LIP is anatomically linked to other saccadic regions, including SC and FEF (Lynch et al., 1985; May, 2006). and thus is a key player in the saccade production circuit. Neurons within LIP neurons are intensely activated by saccades and by saccadic working memory (Barash et al., 1991a; Barash et al., 1991b; Snyder et al., 1997; Thier and Andersen, 1998; Li et al., 2004; Zhang and Barash, 2004; Andersen and Cui, 2009). To examine whether LIP is involved in express saccade generation, we recorded single neuron activity and compared the activity patterns during express and regular saccades. We find that, compared to the no-gap task, 
$\sim 50 \%$ of LIP neurons increase their activity during the gap interval in the gap task. Furthermore, a subset of LIP neurons show enhanced activity during express saccades but not during regular saccades. Our results support the posterior pathway hypothesis in generation of express saccade.

\section{Materials and Methods}

Animal preparation

Two male rhesus monkeys weighing 5-7 $\mathrm{kg}$ were the subjects of the present study. All procedures were approved by the Animal Care Committee of Shanghai Institutes for Biological Sciences, Chinese Academy of Sciences (Shanghai, China). Animals were closely under the supervision of the institute veterinarian daily and during surgery. Under ketamine ( 1 $\mathrm{mg} / \mathrm{kg}$, muscle injection) and $2 \%$ isoflurane ( $\sim 25 \mathrm{ml} / \mathrm{min}$, inhalation) anesthesia, eye coils were implanted under sclera of each eye for measuring eye position (Robinson, 1963), titanium bone screws were screwed into the skull and adhered with acrylic for head fixation, and a $2 \mathrm{~cm}$ diameter recording chamber was implanted at $15 \mathrm{~mm}$ right and $3 \mathrm{~mm}$ posterior for monkey $\mathrm{S}$, and at $15 \mathrm{~mm}$ right and $6 \mathrm{~mm}$ posterior for monkey P from the zero point of the head stereotaxis.

\section{Experimental procedures}

Experiments were carried out in a dark environment. During experiments, monkeys were seated inside a primate chair with their heads firmly fixed to the chair. A 21 inch CRT monitor was placed $55 \mathrm{~cm}$ in front of the monkeys. Visual displays were under the control of a QNX computer running a real-time data acquisition system (REX; NIH, Bethesda, MD). The storage of both neuronal activity and eye position data were under Cerebus (Blackrock Microsystems). Eye movements were recorded with the magnetic search coil technique (Fuchs and Robinson, 1966), which had a resolution of $0.1^{\circ}$. Horizontal and vertical eye position data were collected at $1 \mathrm{kHz}$ frequency. Tungsten microelectrodes $(\sim 1 \mathrm{M} \Omega$ at $1 \mathrm{kHz})$ were lowered through 23 gauge stainless steel guide tubes by an electronic microdrive (NAN Instruments) attached to the recording chambers, and single neuron activity was recorded.

\section{Behavioral paradigms}

Four behavioral tasks were used in the present study, i.e., memory guided saccade, gap saccade, no-gap saccade (step saccade task), and fixation blinking tasks. In the earlier experiments, the target only appeared in one location [neuron's response field (RF)], whereas in the later experiments the target location was increased to two (RF and its opposite location) in some sessions.

Memory-guided saccade task. We used the memory-guided saccade task to find LIP and classify the recorded neurons. Trials began with the appearance of a white cross, the fixation point (FP), on the center of screen. Monkeys were trained to fixate the FP as long as it was on. Following a $500 \mathrm{~ms}$ fixation period within the fixation window, a visual target briefly appeared $(200 \mathrm{~ms})$ at one of eight possible locations, evenly separated and positioned at equal eccentricity $\left(12^{\circ}\right)$. Monkeys had to maintain fixation until fixation point offset, after which they made a single saccade toward the remembered target location. Successful trials were rewarded with a drop of juice.

Gap saccade task. In the one location task, trials began with appearance of a white cross on the center of screen (Fig. 1A). Monkeys were trained to fixate the FP for 500-1000 ms until it was extinguished. Before the target appearance, there was a blank interval (gap) during which the animals had to maintain fixation on the center of the blank screen. Within a block of trials, the gap duration was constant. For monkey $S$, the gap interval was $200-400 \mathrm{~ms}$. For monkey P, the gap interval was $100-200 \mathrm{~ms}$. The gap duration in each block was chosen to produce an SRT distribution with a bimodal profile (Dorris et al., 1997; Schiller et al., 2004b). The position of the visual target was always in a neuron's response field. Monkeys made a saccade as quickly as possible to it (within $500 \mathrm{~ms}$ ) and then maintained fixation for $400 \mathrm{~ms}$ before receiving a reward.

In the two-location task, the target appeared randomly (50/50) at two possible locations, where one was in the neuron's response field and the other was at the same location in the opposite hemifield. Monkeys did not get reward on the error trials. After intensive training, monkeys rarely perform the task wrong (monkey $\mathrm{P}$ had $<1 \%$ error trials, and monkey $\mathrm{S}$ had $4.7 \%$ error trials). The error trials were excluded from the further analysis.

No-gap saccade task. We used the no-gap saccade as a control condition to study the gap effect on neuronal activity. The no-gap saccade task is same as the conventional visually guided saccade task. Trials began with appearance of a white cross on the center of screen. Monkeys were trained to fixate the FP. When the FP disappeared, a visual target appeared simultaneously. Monkeys were required to make a saccade to the target from the initial fixation point.

Fixation blinking task. We employed the fixation blinking task to test whether the gap effect was merely due to the response to fixation offset. Trials began with appearance of a white cross on the center of screen. Monkeys were trained to fixate the FP. The FP vanished for a brief interval, equivalent to the gap interval in gap saccade task. The FP then reappeared at the same location. Monkeys needed to retain their eyes within a $3^{\circ}$ fixation window.

\section{Behavioral data analysis}

Criteria for saccades. The start and end of a saccade was determined by computing the threshold of velocity acceleration and templatematching criteria (Waitzman et al., 1991). We identified the initiation of a saccade when the velocity of eye trace exceeded $20 \%$ s and lasted for $>10 \mathrm{~ms}$. Saccades satisfying the following criteria were analyzed: (1) saccade occurred after the visual target onset (2) saccade duration was $10-200 \mathrm{~ms}$; and (3) saccadic endpoint fell within $5^{\circ}$ window centered at the saccadic target.

Criteria for express and regular saccades. To prevent arbitrarily separating two modes of SRT distribution in gap task, a maximum likelihood estimation was applied to obtain the best fit of the two modes for each monkey (Guan et al., 2012) (Fig. 1B). The intersection point of the two modes of generalized extreme value (GEV) fitting was defined as the boundary between express saccade and regular saccade. For monkey $S$ the boundary was around $100 \mathrm{~ms}$, and for monkey P the boundary was relatively longer, around $124 \mathrm{~ms}$. If SRT was smaller than the boundary, the trial was considered an express saccade; otherwise, it was considered a regular saccade. Trials with SRT $<50 \mathrm{~ms}$ were considered expected saccades and were not included in the present study.

\section{Neuronal data analysis}

Localizing LIP. LIP was localized by its signature single neuron activity, i.e., the persistent activity in a memory-guided saccade task (Barash et al., 1991a; Barash et al., 1991b).

Neuron classification. The recorded neurons were classified into three groups based on their response profile in the memory-guided saccade task. Visual transient response neurons responded to the appearance of visual stimulus, but without presaccadic activity; Saccadic neuron showed either burst or buildup activity rising up during memory interval, and its peak activity correlated with saccade. Persistent response neurons showed sustained activity during the memory interval before the execution of saccades. Since the number of saccadic neurons was few and their activity during gap saccades was similar to that of persistent neurons, we put these two types of neurons together for farther analysis.

Spike density function. The activity of single neurons as well as the population of neurons was presented as a spike density function (SDF). SDFs were generated by substituting each spike with a Gaussian pulse of a specified width $(10 \mathrm{~ms})$ and summing all pulses to produce a continuous function in time.

Activity difference index. The activity difference index (ADI) was calculated by subtracting the baseline activity $(0-100 \mathrm{~ms}$ before gap onset in the gap task, 300-400 ms before visual target onset in the no-gap task) from the activity of a tested interval, for instance the presaccadic interval (50-150 ms before saccade onset), and then the result was divided by the baseline activity.

Activity latency. We used a standard method to compute single neuron response latencies (Bisley et al., 2004). The cutoff of the spike train was calculated by employing the Poisson fit of baseline activity (from $100 \mathrm{~ms}$ before target onset to target onset). The cutoff was the spike number below which $99.9 \%$ spike count would be expected to lie. After stimulus 
A

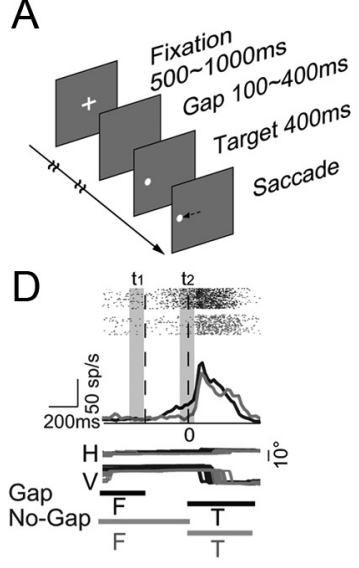

B

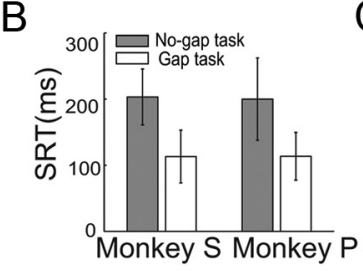

$\mathrm{E}$

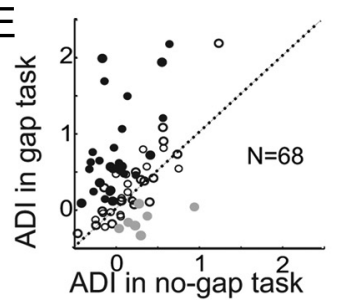

C

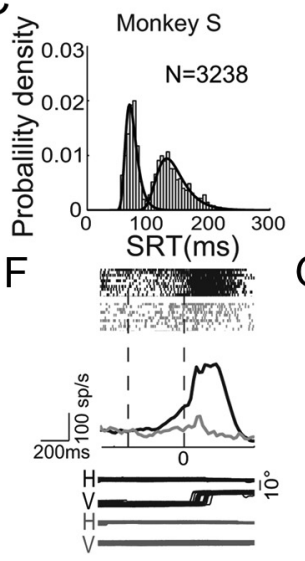

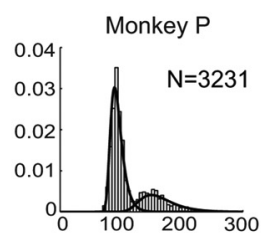

G

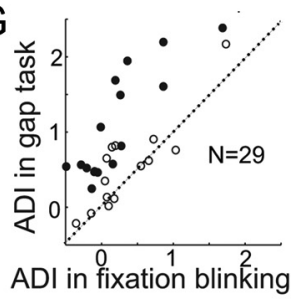

Figure 1. Gap saccade task, monkeys' SRT, and neuronal activity. $\boldsymbol{A}$, Schematic illustration of the gap saccade task. Monkeys are required to maintain fixation on a central fixation point for $500-1000 \mathrm{~ms}$, and then the fixation point turns off. Following a gap interval, a peripheral visual target appears and monkeys are required to make a saccade to it within 500 ms. $\boldsymbol{B}$, Comparison of SRT between no-gap and gap saccade tasks reveals a dramatically shortened SRT in the gap task ( $p \approx 0, t$ test). C, The SRT distributions of both monkeys showed bimodal profiles. A bimodal GEV distribution model was applied to fit the SRT distribution. The boundary of two modes was 100 ms for monkey Sand 124 ms for monkey P. D, The activity of a LIP neuron in the gap and no-gap saccade tasks. Activity is aligned on visual target onset. Eye traces of the two tasks are shown at the bottom ( $\mathrm{H}$, Horizontal eye position; $\mathrm{V}$, vertical eye position; $\mathrm{F}$, fixation point; $T$, visual target). Two dashed vertical lines indicate the gap interval. Two gray patches depict two 100 ms intervals, $t 1$ and $t 2$. This neuron's activity started to gradually increase after fixation offset (first dotted line) in the gap task (dark curve), but not in the no-gap task (gray curve). $\boldsymbol{E}$, Scatter plot showing the comparison of activity difference indexes, ADls, during the gap interval between two tasks for each neuron. Filled dark circles represent neurons with ADI significantly greater in gap task than that in no-gap task $(p<0.05, t$ test). Filled gray circles represent neurons with ADI significantly greater in the no-gap task than that in the gap task ( $p<0.05, t$ test). 0 pen circles indicate neurons with similar ADI in the gap task and no-gap task ( $p>0.05, t$ test). Overall, the population activity was significantly greater in the gap task than that in the no-gap task ( $p=0.0258, t$ test). $F$, An example neuron that responded much more strongly during gap interval in the gap saccade task (dark) than in the fixation blinking task (gray, $p<0.001, t$ test). $G$, The comparison of 29 neurons between gap and fixation blinking task showed that most of them had greater ADI in the gap task; $52 \%$ (15 of 29) had significant stronger activity in gap task (filled circles, $p<0.05, t$ test).

onset, the time of the first of three consecutive bins that contained spikes no less than the cutoff was defined as the response latency.

Correlation coefficient between neuronal activity and SRT. A trial-bytrial correlation coefficient between the neuronal activity $(50 \mathrm{~ms}$ before and $50 \mathrm{~ms}$ after the onset of saccade) and the SRT was calculated for each neuron (Dorris et al., 1997).

\section{Results}

Introducing a gap interval before visual target onset (Fig. $1 A$ ) caused a marked reduction in the mean SRT (by $>80 \mathrm{~ms}$ ) in both monkeys (Fig. $1, B ; p \approx 0, t$ test). Consistent with previous reports (Fischer and Boch, 1983; Fischer and Ramsperger, 1984), saccadic reaction time was distributed bimodally: the first mode consisted of express saccades and the second mode consisted of regular saccades (Fig. 1C). We examined neuronal responses in two intervals: a baseline interval $(t 1$, from -100 $\mathrm{ms}$ to fixation offset in the gap saccade task, left gray patch in Fig. $1 D$, or $300-400 \mathrm{~ms}$ before visual target onset in the nogap task) and a peritarget interval ( $t 2$, from $50 \mathrm{~ms}$ before to 50 $\mathrm{ms}$ after visual target onset, right gray patch in Fig. $1 \mathrm{D}$ ).

\section{Gap causes increased activity in LIP}

We found that many LIP neurons significantly increased their activity in the gap saccade task. Figure $1 D$ superimposes the activity of one such neuron in the gap (black line) and no-gap (gray line) tasks. Eye position and neuronal data are aligned on visual target onset (second dashed vertical line). In the no-gap task, the neuron's discharge remained low until the appearance of visual target, after which the activity rapidly increased. In the gap task, its activity gradually increased following the disappearance of the fixation point (gap interval, between two dashed vertical lines). The neuron's activity during $t 2$ interval was significantly greater in the gap task than that in the no-gap task $\left(p=2.61 \times 10^{-8}, t\right.$ test $)$. To quantify the activity difference, we introduce the "activity difference index" or ADI: the activity difference between $t 2$ and $t 1$ divided by the activity during $t 1$. The ADIs of 68 neurons ( 28 from monkey $\mathrm{P}$ and 40 from monkey $S$ ) are shown in Figure $1 E$. Fifty percent of neurons (34 of 68) had a significant difference between gap and no-gap tasks (filled circles). Of these gap-modulated neurons, 27 of $34(80 \%)$ exhibited enhanced activity in the gap task (dark dots), whereas the remaining 7 neurons (20\%) reduced activity (gray dots). Open circles represent neurons that had no significant difference between gap and no gap task. Overall, paired $t$ test of the recorded population revealed that the activity in the gap task was significantly greater than that in the no-gap task $(p=0.0082)$.

\section{Gap effect is not due to the physical offset of fixation point}

To test whether the gap effect was due to the physical offset of the fixation point, we compared the neuronal activity between gap saccade task and fixation blinking task. In the fixation blinking task, the fixation point turned off briefly and then came on again (blank interval, same duration as the gap interval); however, no peripheral visual target appeared. Monkeys were trained to maintain fixation throughout the trial, even when the fixation spot was absent. As shown in Figure $1 F$, there is markedly stronger activity in the gap saccade task (black trace) than in the fixation blinking task (gray trace). The activity difference during the gap interval between these two tasks is highly significant $\left(p=2.54 \times 10^{-9}, t\right.$ test). The results of comparison between gap and blinking task for 29 neurons ( 13 from monkey $\mathrm{P}$ and 16 from monkey S) are presented in Figure $1 G$. None of the neurons show significantly stronger activity in the fixation blinking task than in the gap task. Paired $t$ test of the population data shows significantly greater activity in gap saccade task than that in fixation blinking task $(p=0.0007)$, indicating that presaccadic enhancement is not simply due to fixation point offset. 
A

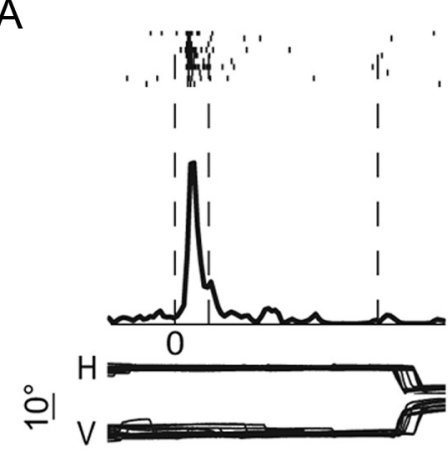

$\mathrm{B}$

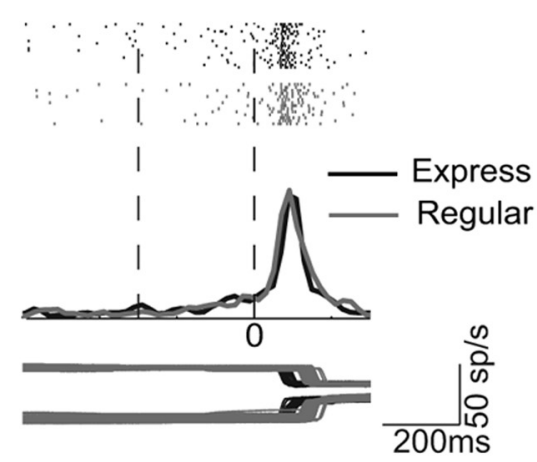

E
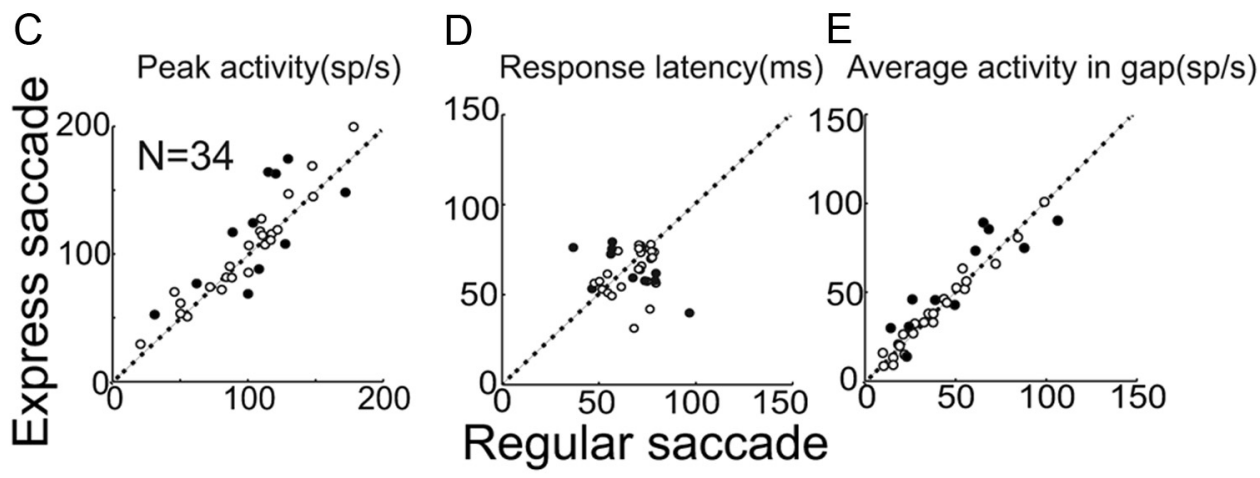

Figure 2. Activity of visual neurons in the gap saccade task. $\boldsymbol{A}$, The response of an example neuron in memory-guided saccades is closely correlated with the appearance of visual stimulus. Three vertical dashed lines indicate target onset, target offset, and fixation offset, respectively. $\boldsymbol{B}$, Comparison of this neuron's activity in gap task between express and regular saccades shows that there is no significant difference $(p=0.32, t$ test). Two vertical dashed lines depict fixation offset and target onset. $\boldsymbol{C}-\boldsymbol{E}$, The comparison of visual neurons between express and regular saccades on three aspects: peak activity $(\boldsymbol{C}$, response latency $(\boldsymbol{D})$, and average activity $(\boldsymbol{E})$ in gap interval. Filled circles depict that the activity difference is statistically significant $(p<0.05$, paired $t$ test), whereas the open circles indicate that the activity is similar ( $p>0.05$, paired $t$ test). Overall, population data show no significant difference (the smallest, $p=0.57$, paired $t$ test).

Visual neurons do not show express saccade-related activity It has been proposed that the express saccades are generated by the facilitation of visual processing (Reulen, 1984). If this is the case, we would expect to see an early evoked or more vigorous visual response during express saccades. However, recorded visual neurons did not show significantly enhanced activity between express and regular saccades. The activity of an example visual neuron in memory-guided saccades and gap saccades are presented in Figure 2, $A$ and $B$, respectively. In memory-guided saccades, this neuron shows transient burst discharge following visual target onset but lacks persistent activity (Fig. $2 A$ ). In the gap task, its activity in express saccade trials (black) is similar to that in regular saccade trials (gray) ( $p=0.10, t$ test, Fig. $2 B$ ). This observation is true for the population data. The comparisons of 34 visual neurons (14 from monkey $\mathrm{P}$ and 20 from monkey S) between gap and no-gap trials do not differ regarding visual peak activity (Fig. 2C), visual response latency (Fig. 2D), and average activity (Fig. 2E) during gap interval. Even though a few data points show significant difference (filled circles), most of them are distributed along the diagonal line (open circles). Overall, the population data do not show any significant difference between express and regular saccades ( $p>0.5$ for peak activity; $p>0.7$ for response latency; $p>0.7$ for averaged activity, paired $t$ test). Such results do not support the visual facilitation hypothesis.

\section{A group of persistent neurons show enhanced activity during express saccades}

In contrast to visual neurons, some persistent neurons show significantly greater activity in express saccades than that in regular saccades. As an example, the neuron shown in Figure $3 A$ has persistent response during memory-guided saccades.
In the gap task (Fig. $3 B$ ), this neuron fires more strongly throughout the fixation and gap periods in express saccade trials (black) than that in regular saccade trials (gray) $(p=$ $2.73 \times 10^{-5}$ for fixation interval; $p=3.90 \times 10^{-5}$ for gap interval; $p=1.25 \times 10^{-5}$ for presaccadic interval, $t$ test) and reaches peak response earlier. Since the enhanced activity emerged very early in the trial, the higher activity in express saccades presumably reflects top-down modulation, such as attention (Currie et al., 1993; Munoz et al., 2003; Bisley and Goldberg, 2006; Guan et al., 2012) or motor preparation (Dorris et al., 1997; Everling and Munoz, 2000). Moreover, the neuron's activity reaches the peak immediately before the execution of saccadic eye movement (red markers in raster plots) in both express and regular saccade trials, indicating the close relationship between neuronal activity and the generation of saccades.

However, not all of the persistent neurons have enhanced activity in express saccades. Figure $3 C, D$ shows another persistent neuron in memory-guided saccades which, in contrast, exhibits decreased activity in the express saccade task relative to that in the regular saccade task. About half of the persistent neurons do not show significantly different activity between express and regular saccades (Fig. $3 E, F$ ). We call the first two types express saccade-related neurons and the third type express saccade-unrelated persistent neurons. We compared the activity of 82 neurons ( 35 from monkey P and 47 from monkey $S$ ) between express and regular saccades during $t 2$ interval to find out how many persistent neurons are express saccaderelated (Fig. 4A). We found 28 of 82 (34\%) showed statistically significant increase of activity in express saccades (express saccade-enhanced persistent neuron, filled dark circles), 9 of 
A

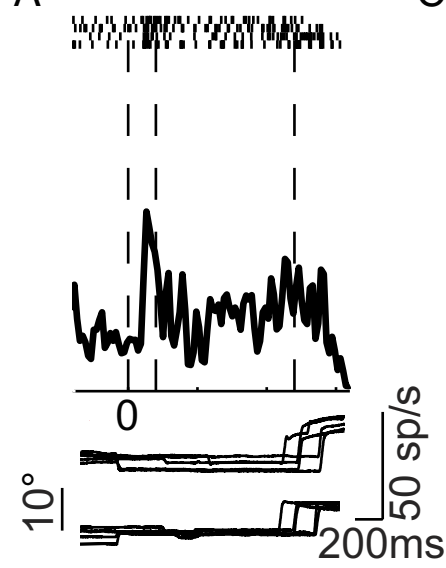

B

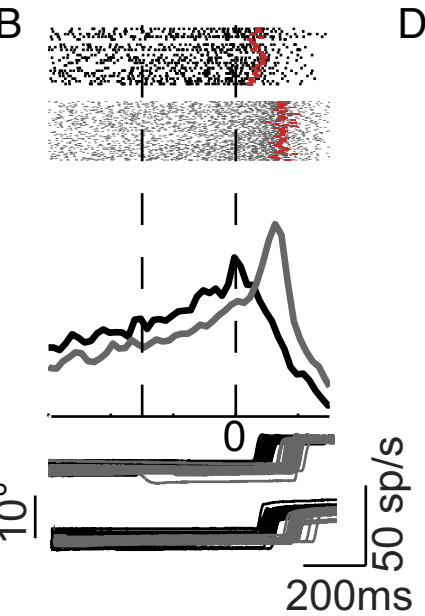

C
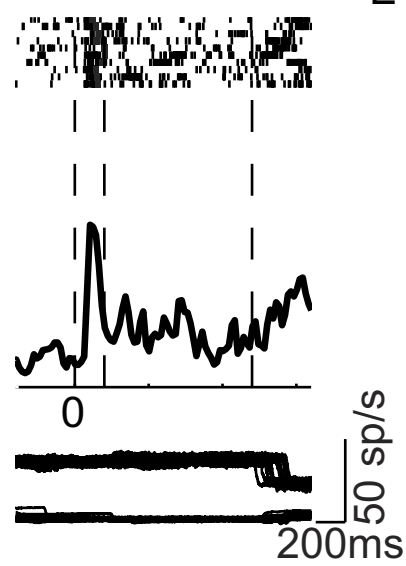

E
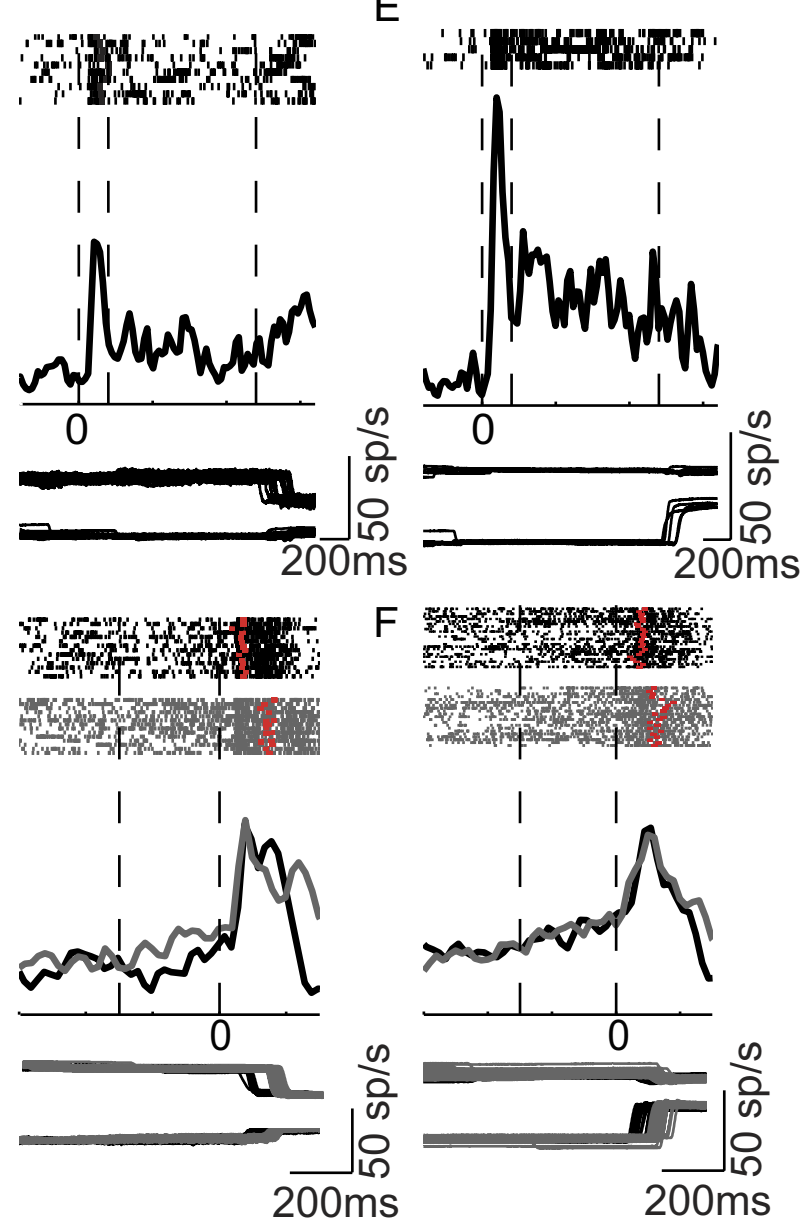

$F$ w

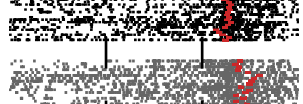

竞
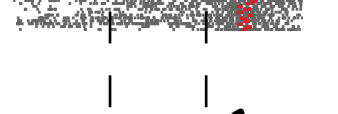

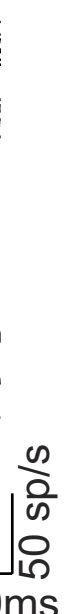

Figure 3. Activity of example persistent neurons in the gap saccade task. $\boldsymbol{A}$, Activity in memory-guided saccade task shows persistent activity. $\boldsymbol{B}$, When the activity in the gap saccade task is aligned on visual target onset (second dashed line), this neuron shows significantly greater activity during express saccade (dark line) than during regular saccade (gray line) $\left(p=2.73 \times 10^{-5}\right.$ for initial fixation; $p=3.90 \times 10^{-5}$ for gap interval; $p=1.25 \times 10^{-5}$ for presaccadic interval; $t$ test), and its peak activity is earlier in express saccades. Red ticks denote onset of saccades. $\boldsymbol{C}$, Another persistent neuron in memory-guided saccade. $\boldsymbol{D}$, The neuron's activity is lower during express saccades compared with that during regular saccades. $\boldsymbol{E}$, The third persistent neuron in memory-guided saccade. $\boldsymbol{F}$, This neuron responds similarly between express and regular saccades ( $p=0.12, t$ test).

$82(11 \%)$ showed statistically significant decrease of activity in express saccades (express saccade-suppressed persistent neuron, filled gray circles), and 45 of 82 (55\%) showed no significant difference (express saccade-unrelated persistent neuron, open circles). The population activity of 28 express saccade-enhanced persistent neurons is shown in Figure $4 B$ (95\% confidence interval indicated by dotted lines), the population activity of 9 express saccade-suppressed persistent neurons is shown in Figure $4 C$, and the population activity of 45 express saccade-unrelated persistent neurons is shown in Figure $4 D$. For express saccadeenhanced persistent neurons, the population activity from initial fixation to shortly after target onset is significantly stronger ( $p=$ $9.22 \times 10^{-6}, t$ test) and peak time is earlier in express saccades than regular saccades. In contrast, nine express saccadesuppressed persistent neurons show significantly decreased activity $(p=0.0014, t$ test $)$ in express saccades comparing with regular saccades during gap interval. For express saccade-unrelated persistent neurons, the population activity does not differ between express and regular saccades $(p=0.30, t$ test $)$.

A group of persistent neurons' activity closely relates with SRT

To further test whether and how the enhanced activity may correlate with generating express saccades, we analyzed the trial by trial relationship between neuronal activity and SRT in intervals $t 1$ and $t 2$. The correlation coefficients between each single neuron's spike rate and SRT were calculated by using linear regression analysis. Figure 5 shows examples of the activity-SRT correlation coefficients of a visual neuron (Fig. $5 A$ ), an express saccade-unrelated persistent neuron (Fig. 5B), and an express saccade-enhanced persistent neuron (Fig. 5C). Regression fitting of the data reveals that there are no significant correlations between discharge rate and SRT in $t 1$ and $t 2$ intervals for both visual and express saccade-unrelated persistent neurons. The significant correlation exists only for the express saccade-enhanced persistent neurons and is significant in both $t 1$ and $t 2$ intervals $(r=-0.2459$ and -0.4348$)$. The negative value of $r$ indicates that higher spiking rate correlates with shorter SRT.

This relationship can also be seen in the cumulative percentage distributions of the activity-SRT correlation coefficients. In activity-SRT correlation coefficient analysis, the closer the distribution is to 0 , the lower the correlation is. While the mean of cumulative percentage distribution of visual neuron (Fig. 6A) and express saccade-unrelated persistent neurons (Fig. 6B) do not show significant difference from 0 (the smallest, $p=0.14$ ), the express saccade-enhanced persistent neurons (Fig. $6 C$ ) show significant leftward shifts of cumulative percentage distribution in both $t 1$ (dark gray) $(p=0.04)$ and $t_{2}$ (light gray, $p=5.58 \times$ 
A

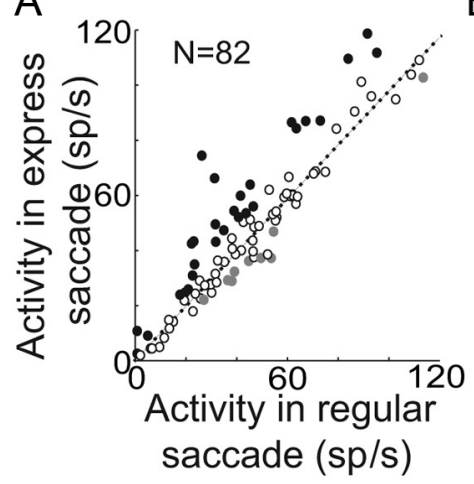

B

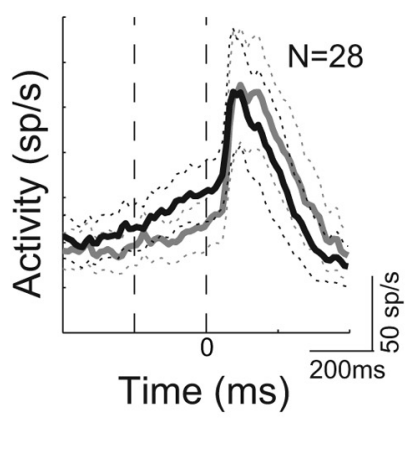

C

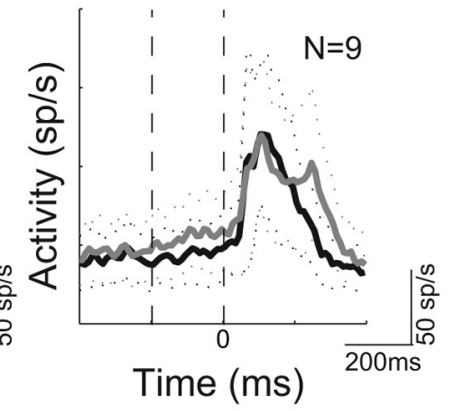

$\mathrm{D}$

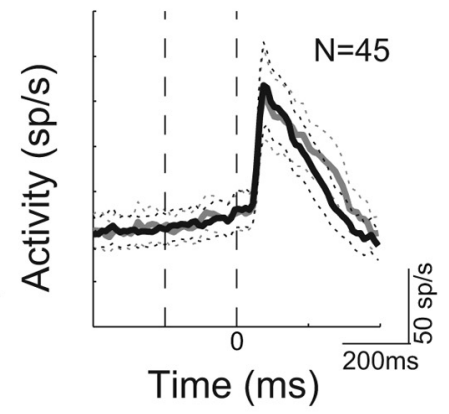

Figure 4. Population activity of persistent neurons in gap saccade task. $\boldsymbol{A}$, The comparison of presaccadic activity between express and regular saccades. Dark dots, Neurons with stronger activity in express saccades; gray dots, neurons with decreased activity in express saccades; open circles, neurons with no difference between express and regular saccades. $\boldsymbol{B}$, Population response of 28 express saccade-enhanced persistent neurons with $95 \%$ confident interval. These neurons show stronger activity and earlier peak during express saccades $\left(p=9.22 \times 10^{-6}, t\right.$ test). $C$, Population response of nine express saccadesuppressed persistent neurons shows decreased activity $(p=0.0014, t$ test) during express saccades. $\boldsymbol{D}$, Population response of express saccade-unrelated persistent neurons show no significant difference between express and regular saccades ( $p=0.30, t$ test).

$\left.10^{-8}\right)$. The larger shift in $t 2$ indicates a stronger negative correlation of activity-SRT in the $t 2$ interval. The existence of significant relationship between neuronal activity and SRT indicates that the enhanced activity in LIP plays an important role in saccade generation.

\section{Target uncertainty affects both SRT and the neuronal activity in LIP}

To examine whether the enhanced express saccade activity might be driven by spatial uncertainty, we reduced spatial certainty by adding second possible target location in gap saccade task. This second target was located in the opposite hemifield at the same eccentricity with 50/50 distribution. We recorded 37 persistent neurons in both one location (one-loc) and two locations (two-loc) tasks. Our working hypothesis is that monkeys can generate express saccades in the two-loc task whether the anticipated location is same as the actual location of the target (valid anticipation); but, if the anticipated location differs from the location of the target (invalid anticipation), the SRT will be longer than that in the one-loc task, as additional time is needed for attention/motor preparation shifting from unmatched location to the target location. Consistent with our expectation, the SRT distributions (Fig. 7A) of two monkeys in the two-loc task show lower express saccade rates and prolonged SRT in regular saccades comparing with one-loc task. Notably, the mean SRT of express saccades does not differ between one-loc and two-loc conditions ( $p=$ 0.0954 for monkey $\mathrm{S} ; p=0.0628$ for monkey $\mathrm{P}, t$ test).

The change of neuronal activity in LIP is highly correlated with the change of SRT. Figure $7 B$ illustrates the activity of an example neuron in the one-loc and two-loc tasks when the target is in its response field, RF, and a saccade is directed to it. The neuron's response is similar during express saccades regardless of the certainty (blue for express saccades in one-loc, black for express saccades in two-loc, $p=0.3216, t$ test). In contrast, a marked activity difference occurs between one-loc and two-loc tasks during regular saccades (purple for regular saccade in one-loc task, gray for regular saccade in two-loc task, $p=0.0096, t$ text), reflecting the modulation of spatial anticipation for saccadic target. For trials in which the target appears in and the saccade directed to the null direction (opposite of the RF), the neuron's activity also reveals anticipation modulation. As shown in Figure $7 C$, in regular saccade trials the neuron's activity during the gap interval is higher (gray) than that in express saccade trials (black) $(p=0.0077$, $t$ test), which indicates anticipation being in null direction. These observations are supported by population data (37 neurons). Within RF trials, the activity in express saccade trials is similar between one-loc and two-loc task (Fig. $8 A, p=0.3485$, paired $t$ test), and the activity in regular saccade trials is significantly biased to one-loc (Fig. $8 B, p=0.0043$, paired $t$ test). In opposite RF trials, the activity in regular saccade trials is significantly greater than that in express saccade trials (Fig. 8 $C, p=4.2018^{\star} 10-6$, paired $t$ test $)$.

\section{Discussion}

In the present study, we try to define whether and under what conditions LIP neurons are activated when the monkey performs saccades. In particular, we focus on comparison of trials in which the SRT is in the express range (for monkey S, SRT = $50-100 \mathrm{~ms}$; for monkey P, SRT $=50-124 \mathrm{~ms}$ ) to trials in which the SRT of the saccade is in the regular range (for monkey S, SRT > $100 \mathrm{~ms}$; for monkey P, SRT > $124 \mathrm{~ms}$ ). We do not address here some issues raised in the express saccade literature, such as the role of selective attention in express saccade generation (Mayfrank et al., 1986; Fischer and Breitmeyer, 1987; Mackeben and Nakayama, 1993; Dias and Bruce, 1994; Schiller et al., 2004a; Guan et al., 2012). Here, we have focused only on whether LIP contains activity related to express saccades.

To address this question, we examined how a gap interval affects the neuronal activity in LIP of rhesus monkeys. We found that about half the recorded LIP neurons were modulated by the gap interval-with stronger pretarget activity in the gap saccade task than that in the no-gap saccade task. Similar gap modulation has been observed in FEF (Dias and Bruce, 1994; Everling and Munoz, 2000; Sommer and Wurtz, 2000), and SC (Munoz and Wurtz, 1992; Dias and Bruce, 1994; Edelman and Keller, 1996; Dorris et al., 1997; Edelman and Keller, 1998; Everling and Munoz, 2000; Sommer and Wurtz, 2000). We show that the increased activity was not simply due to the physical disappearance of fixation point. Furthermore, we find that a subgroup of persistent neurons show enhanced activity in express saccade trials from the beginning of initial fixation until target onset. Uncertainty of target location markedly affects monkeys' SRT through modulating the pre- 

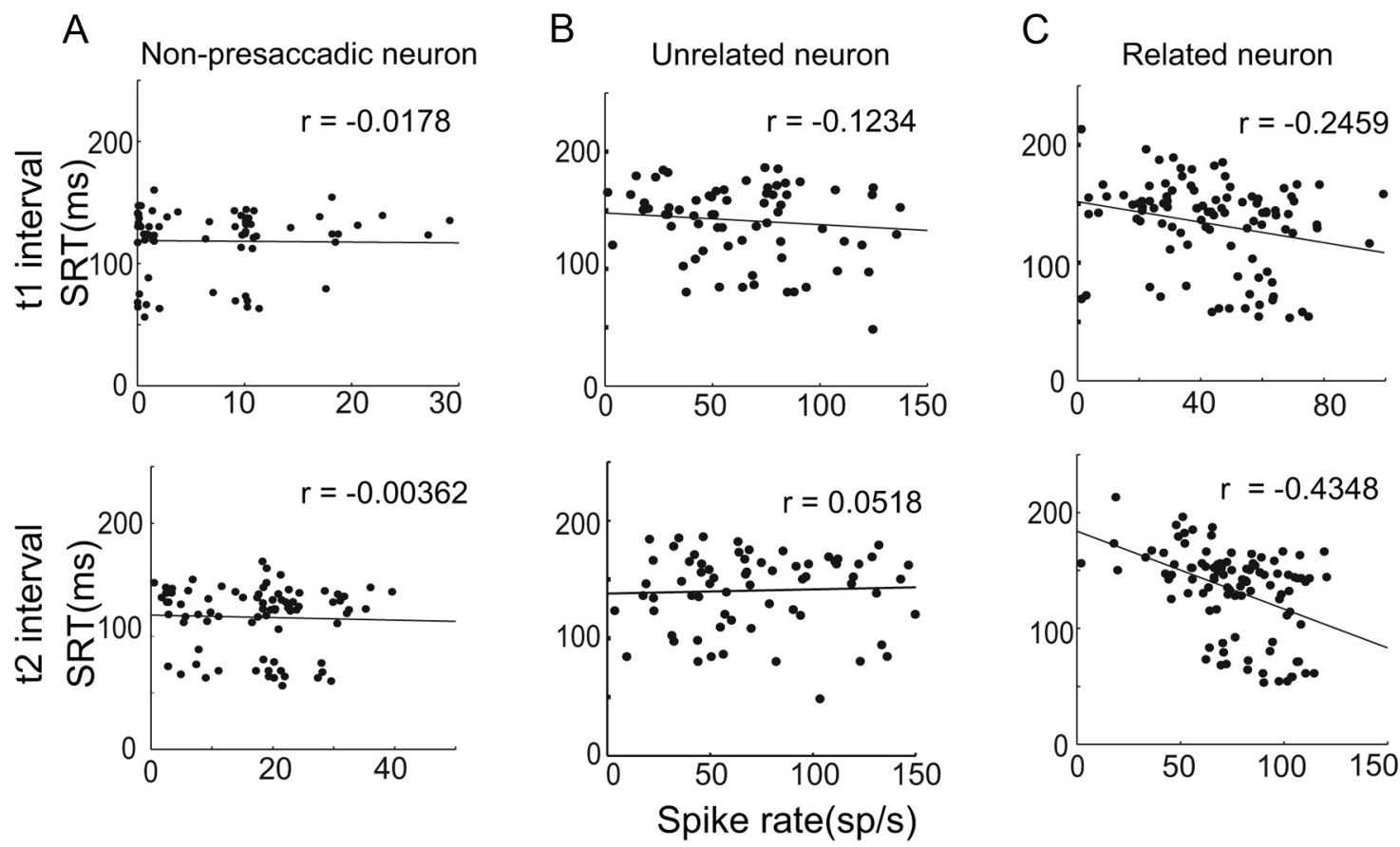

Figure 5. Correlations between activity and SRT. $\boldsymbol{A}-\boldsymbol{C}$, Correlation coefficients between neuronal activity (in two intervals: $t 1$ and $t 2)$ and SRT: a visual neuron $(\boldsymbol{A})$, an express saccade-unrelated persistent neuron $(\boldsymbol{B})$, and an express saccade-enhanced persistent neuron $(\boldsymbol{C})$. In both $t 1$ and $t 2$ intervals there are no significant correlations for visual neuron $(r=$ -0.0178 and -0.0362$)$ and for express saccade-unrelated persistent neuron $(r=-0.1234$ and 0.0518$)$. Only express saccade-enhanced persistent neurons exhibit significant correlation in both intervals $(t 1, r=-0.2459 ; t 2, r=-0.4348)$.

paratory activity of LIP neurons. The significantly reduced activity in regular saccade in two-loc task reflects the negative modulation of anticipation from the opposite location of the RF (null direction). The results provide the first electrophysiological evidence for the involvement of LIP in the generation of express saccades. Pretarget preparatory activity might play a role in determining the SRT.

\section{Two distinct effects of gap interval in LIP}

The presence of the gap during goaldirected saccade tasks affects the activity of LIP in two ways. First, it leads to increased activity during the gap interval (general modulation) regardless of whether saccades are express or regular mode. Second, specifically enhanced activity (express saccadeenhanced activity) occurs only during express saccade trials.

While general gap modulation had been reported in the FEF and SC (Munoz and Wurtz, 1992; Dias and Bruce, 1994; Edelman and Keller, 1996; Dorris et al., 1997; Edelman and Keller, 1998; Everling and Munoz, 2000; Sommer and Wurtz, 2000), express saccade-enhanced activity has only been reported in SC (Dorris et al., 1997). The fact that express saccade-related enhancement was not found in FEF (Dias and Bruce, 1994; Everling and Munoz, 2000) indicates distinct roles of these three saccadic centers in saccadic generation. As there are direct projections from LIP to the intermediate and deep layers of SC (Lynch et al., 1985; May, 2006) and indirect projections from the intermediate and deep layer of SC to LIP via thalamus and FEF (May, 2006), express saccade-related activity in LIP is unlikely to be driven $t 1$ and $t 2$ interval.
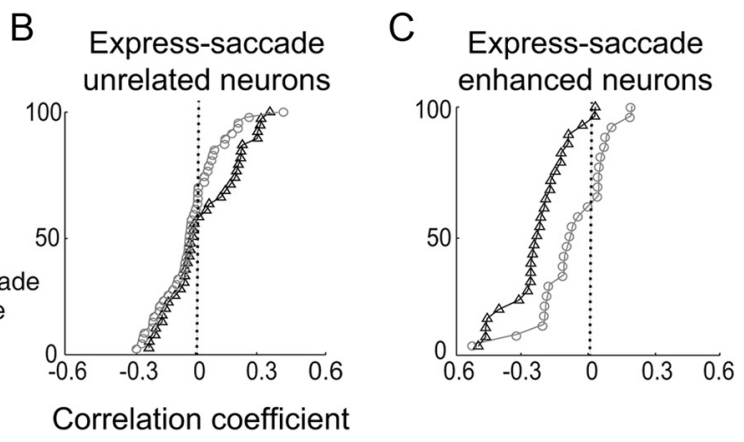

Figure 6. Cumulative percentage distribution of the activity-SRT correlation coefficients. $A, B$, The cumulative percentage distribution of visual neurons $(\boldsymbol{A})$ and express saccade-unrelated persistent neurons $(\boldsymbol{B})$ are centered near 0 . $\boldsymbol{C}$, The cumulative percentage distribution of express saccade-enhanced persistent neurons is significantly shifted to left in both

by SC. Rather, LIP is likely to drive express saccade related activity in SC.

The gap effect was suggested to be a neuronal correlate to "fixation disengagement" (Dias and Bruce, 1994). Interestingly, we found that the fixation disengagement in LIP was not a simple response to the physical disappearance of FP (Fig. 1 $F)$; rather, it might reflect cognitive modulation such as attention (Currie et al., 1993; Munoz et al., 2003; Bisley and Goldberg, 2006; Guan et al., 2012) or motor preparation (Dorris et al., 1997; Everling and Munoz, 2000).

\section{Preparatory activity plays crucial role in determining SRT}

It had been proposed that both the preparatory activity before visual target onset and visual burst activity following target onset play a crucial role in express saccade generation (Paré and Munoz, 1996; Dorris et al., 1997; Sparks et al., 2000; Boehnke and Munoz, 2008). In the present study, we found 
A

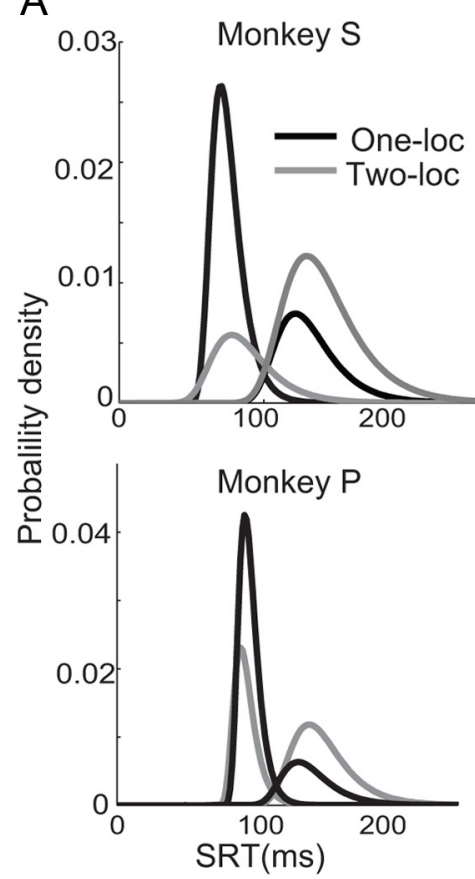

B

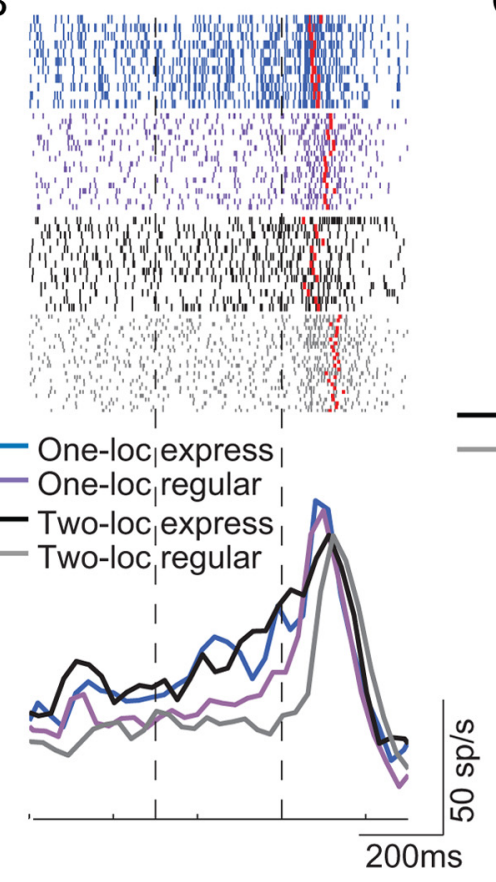

C

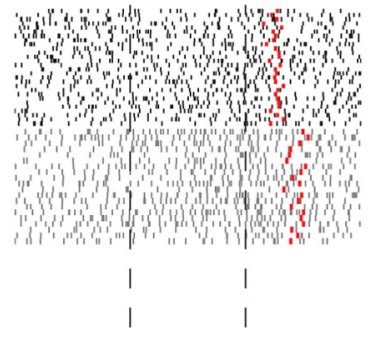

Two-loc express

Two-loc regularl

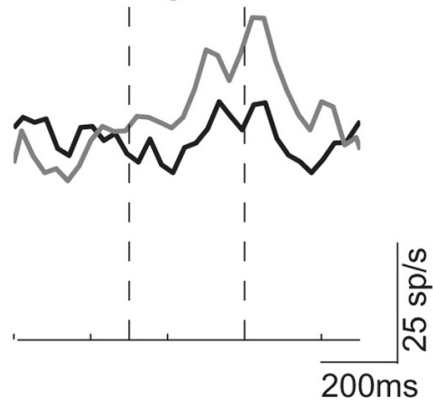

Figure 7. SRT and single neuronal activity in one-loc and two-loc tasks. A, SRT distributions of two monkeys show lower proportion of express saccades and more discomposed distribution of regular saccades in two-loc (gray) compared with that in one-loc (black). While the SRT distributions of express saccades remain similar between one-loc and two-loc trials ( $p=0.0954$ for monkey $S, p=0.0628$ for monkey $\mathrm{P}, t$ test), the SRT distribution in regular saccades is decomposed in two-loc trials rather than in one-loc trials $(p \approx 0$ for both monkeys, $t$ test). $B$, Shown is an example of neuron's activity in one-loc and two-loc trials when target is in and saccade directs to neuron's RF. Two dashed vertical lines denote the gap interval. The activity is similar in express saccade trials between one-loc trials (blue) and two-loc trials (dark, $p=0.3216, t$ test); the activity in the regular saccade trials of two-loc task (gray) is significantly lower than that in the regular saccade trials of one-loc task (purple, $p=0.0096, t$ test). $C$, The same neuron's activity in two-loc task when target is in and saccade directs to the null direction. The activity is higher during gap interval in regular saccade trials (gray) than in express trials (dark, $p=0.0077, t$ test).
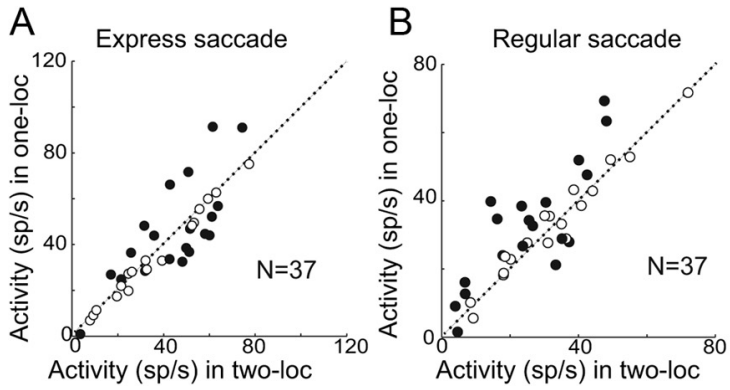

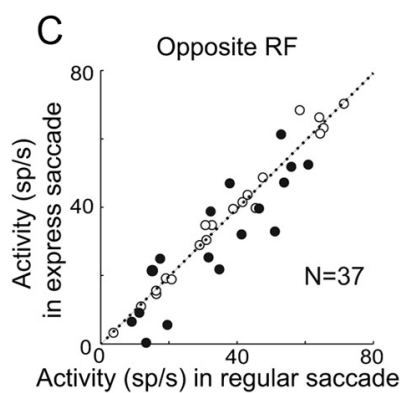

Figure 8. Comparison of population activity between one-loc and two-loc trials. $A$, Within RF trials, the activity comparison of population neurons during express saccades between one-loc and two-loc tasks does not show significant difference ( $p=0.35$, paired $t$ test). $\boldsymbol{B}$, Conversely, the population activity during regular saccades shows significant stronger activity in one-loc task ( $p=0.004$, paired $t$ test). $C$, In opposite RF trials, the comparison of population activity between express and regular saccades in two-loc task shows greater activity in regular saccades $\left(p=4.20 \times 10^{-6}\right.$, paired $t$ test). Filled black dots show that the activity difference of signal neurons under two conditions is statistically significant $(p<0.05, t$ test) whereas opened circles are not.

that visual neurons did not show any significant difference in response latency, peak activity, or averaged pretarget activity (Fig. 3). Instead, a group of persistent neurons increased activity from the beginning of initial fixation until target onset during express saccade trials compared with regular saccade trials (Fig. 4 and Fig. 5). Such increase is spatially selective and is limited to the neuron's response field. Our results indicate that the enhanced preparatory activity plays crucial role in determination of the SRT.

The preparatory activity in LIP seems to scale with the probability of making a saccade as SC neurons do (Basso and Wurtz, 1998; Dorris and Munoz, 1998). Increase of spatial un- certainty might increase the competition of coexisting motor plans. As a result, increased spatial uncertainty significantly decreases the possibility of making express saccade. But, if the preparatory activity reaches the threshold level, a saccade will be triggered (similar activity during express saccades in both one-loc and twoloc conditions; Fig. 7).

The existence of express saccadeenhanced persistent neurons in LIP supports the findings from lesion study of posterior parietal cortex in human and nonhuman primates in which the number of express saccades decreased and the SRT distribution of regular saccade shifted to the right (Lynch and McLaren, 1989; Braun et al., 1992; Li et al., 1999). Our results also support the findings of a more recent human EEG study in which authors found the increased activity in occipital-parietal network during mid-gap and lategap intervals preceding express saccades (Hamm et al., 2010).

\section{LIP processes visual and saccadic information in the early epoch of behavioral tasks}

Unlike the lower visual cortical regions in humans and rhesus monkeys, LIP is a node linking visual and saccadic circuitry together. Accumulating evidence has indicated that LIP is involved in processing visual, saccadic, and cognitive signals (Barash et al., 1991a; Barash et al., 1991b; Snyder et al., 1997; Thier and Andersen, 1998; Colby and Goldberg, 1999; Bisley 
and Goldberg, 2003; Li et al., 2004; Andersen and Cui, 2009; , 2010; Guan et al., 2012) (Platt and Glimcher, 1999; Shadlen and Newsome, 2001; Gold and Shadlen, 2007; Ganguli et al., 2008; Kiani and Shadlen, 2009). LIP neurons often show strong task-dependent activity, i.e., they activate very differently in different behavioral tasks. For instance, in a previous study we found that some visual neurons expressed vigorous "paradoxical activity" in memory antisaccade, i.e., its timing was visual-like but its direction was motor-like (Zhang and Barash, 2000). We believe that the paradoxical activity plays a crucial role in facilitating the inversion visuomotor transformation during antisaccade. Another example is visual receptive field remapping across saccadic eye movement (Duhamel et al., 1992). About two-thirds of visual neurons in LIP shift their receptive fields to cover the future receptive field before or soon after the initiation of saccades. Such remapping activity is important for fast updating of retinotopic information to create a stabilized visual world across saccades. Express saccade related activity, paradoxical activity, and visual remapping activity all show transient burst discharge activity immediately following the appearance of the visual stimulus but are generated by different types of neurons. The paradoxical activity and the visual remapping activity are produced by primarily visual response neurons, whereas express saccaderelated activity is found primarily in saccadic response neurons. Such results indicate that LIP not only processes visual information in the earlier epoch of behavioral task but also deals with saccadic information.

\section{References}

Andersen RA, Cui H (2009) Intention, action planning, and decision making in parietal-frontal circuits. Neuron 63:568-583. CrossRef Medline

Barash S, Bracewell RM, Fogassi L, Gnadt JW, Andersen RA (1991a) Saccade-related activity in the lateral intraparietal area. I. Temporal properties; comparison with area 7a. J Neurophysiol 66:1095-1108. Medline

Barash S, Bracewell RM, Fogassi L, Gnadt JW, Andersen RA (1991b) Saccade-related activity in the lateral intraparietal area. II. Spatial properties. J Neurophysiol 66:1109-1124. Medline

Basso MA, Wurtz RH (1998) Modulation of neuronal activity in superior colliculus by changes in target probability. J Neurosci 18:7519-7534. Medline

Bisley JW, Goldberg ME (2003) Neuronal activity in the lateral intraparietal area and spatial attention. Science 299:81-86. CrossRef Medline

Bisley JW, Goldberg ME (2006) Neural correlates of attention and distractibility in the lateral intraparietal area. J Neurophysiol 95:1696-1717. CrossRef Medline

Bisley JW, Goldberg ME (2010) Attention, intention, and priority in the parietal lobe. Annu Rev Neurosci 33:1-21. CrossRef Medline

Bisley JW, Krishna BS, Goldberg ME (2004) A rapid and precise onresponse in posterior parietal cortex. J Neurosci 24:1833-1838. CrossRef Medline

Boehnke SE, Munoz DP (2008) On the importance of the transient visual response in the superior colliculus. Curr Opin Neurobiol 18:544-551. CrossRef Medline

Braun D, Weber H, Mergner T, Schulte-Mönting J (1992) Saccadic reaction times in patients with frontal and parietal lesions. Brain 115:1359-1386. CrossRef Medline

Colby CL, Goldberg ME (1999) Space and attention in parietal cortex. Annu Rev Neurosci 22:319-349. CrossRef Medline

Currie J, Joyce S, Maruff P, Ramsden B, McArthur-Jackson C, Malone V (1993) Selective impairment of express saccade generation in patients with schizophrenia. Exp Brain Res 97:343-348. Medline

Dias EC, Bruce CJ (1994) Physiological correlate of fixation disengagement in the primate's frontal eye field. J Neurophysiol 72:2532-2537. Medline

Dorris MC, Munoz DP (1998) Saccadic probability influences motor preparation signals and time to saccadic initiation. J Neurosci 18:7015-7026. Medline

Dorris MC, Paré M, Munoz DP (1997) Neuronal activity in monkey supe- rior colliculus related to the initiation of saccadic eye movements. J Neurosci 17:8566-8579. Medline

Duhamel JR, Colby CL, Goldberg ME (1992) The updating of the representation of visual space in parietal cortex by intended eye movements. Science 255:90-92. CrossRef Medline

Edelman JA, Keller EL (1996) Activity of visuomotor burst neurons in the superior colliculus accompanying express saccades. J Neurophysiol 76: 908-926. Medline

Edelman JA, Keller EL (1998) Dependence on target configuration of express saccade-related activity in the primate superior colliculus. J Neurophysiol 80:1407-1426. Medline

Everling S, Munoz DP (2000) Neuronal correlates for preparatory set associated with pro-saccades and anti-saccades in the primate frontal eye field. J Neurosci 20:387-400. Medline

Fischer B, Boch R (1983) Saccadic ey movements after extremely short reaction times in the monkey. Brain Res 260:21-26. CrossRef Medline

Fischer B, Breitmeyer B (1987) Mechanisms of visual attention revealed by saccadic eye movements. Neuropsychologia 25:73-83. CrossRef Medline

Fischer B, Ramsperger E (1984) Human express saccades: extremely short reaction times of goal directed eye movements. Exp Brain Res 57:191-195. Medline

Fuchs AF, Robinson DA (1966) A method for measuring horizontal and vertical eye movement chronically in the monkey. J Appl Physiol 21: 1068-1070. Medline

Ganguli S, Bisley JW, Roitman JD, Shadlen MN, Goldberg ME, Miller KD (2008) One-dimensional dynamics of attention and decision making in LIP. Neuron 58:15-25. CrossRef Medline

Gold JI, Shadlen MN (2007) The neural basis of decision making. Annu Rev Neurosci 30:535-574. CrossRef Medline

Guan S, Liu Y, Xia R, Zhang M (2012) Covert attention regulates saccadic reaction time by routing between different visual-oculomotor pathways. J Neurophysiol 107:1748-1755. CrossRef Medline

Hamm JP, Dyckman KA, Ethridge LE, McDowell JE, Clementz BA (2010) Preparatory activations across a distributed cortical network determine production of express saccades in humans. J Neurosci 30:7350-7357. CrossRef Medline

Isa $\mathrm{T}$ (2002) Intrinsic processing in the mammalian superior colliculus. Curr Opin Neurobiol 12:668-677. CrossRef Medline

Kiani R, Shadlen MN (2009) Representation of confidence associated with a decision by neurons in the parietal cortex. Science 324:759-764. CrossRef Medline

Li CS, Mazzoni P, Andersen RA (1999) Effect of reversible inactivation of macaque lateral intraparietal area on visual and memory saccades. J Neurophysiol 81:1827-1838. Medline

Li ZH, Sun XW, Wang ZX, Zhang XC, Zhang DR, He S, Hu XP (2004) Behavioral and functional MRI study of attention shift in human verbal working memory. Neuroimage 21:181-191. CrossRef Medline

Lynch JC, McLaren JW (1989) Deficits of visual attention and saccadic eye movements after lesions of parietooccipital cortex in monkeys. J Neurophysiol 61:74-90. Medline

Lynch JC, Graybiel AM, Lobeck LJ (1985) The differential projection of two cytoarchitectonic subregions of the inferior parietal lobule of macaque upon the deep layers of the superior colliculus. J Comp Neurol 235:241254. CrossRef Medline

Mackeben M, Nakayama K (1993) Express attentional shifts. Vision Res 33:85-90. CrossRef Medline

May PJ (2006) The mammalian superior colliculus: laminar structure and connections. Prog Brain Res 151:321-378. CrossRef Medline

Mayfrank L, Mobashery M, Kimmig H, Fischer B (1986) The role of fixation and visual attention in the occurrence of express saccades in man. Eur Arch Psychiatry Neurol Sci 235:269-275. CrossRef Medline

Munoz DP, Wurtz RH (1992) Role of the rostral superior colliculus in active visual fixation and execution of express saccades. J Neurophysiol 67: 1000-1002. Medline

Munoz DP, Armstrong IT, Hampton KA, Moore KD (2003) Altered control of visual fixation and saccadic eye movements in attention-deficit hyperactivity disorder. J Neurophysiol 90:503-514. CrossRef Medline

Paré M, Munoz DP (1996) Saccadic reaction time in the monkey: advanced preparation of oculomotor programs is primarily responsible for express saccade occurrence. J Neurophysiol 76:3666-3681. Medline

Platt ML, Glimcher PW (1999) Neural correlates of decision variables in parietal cortex. Nature 400:233-238. CrossRef Medline 
Reulen JP (1984) Latency of visually evoked saccadic eye movements. I. Saccadic latency and the facilitation model. Biol Cybern 50:251-262. CrossRef Medline

Robinson DA (1963) A method of measuring eye movement using a scleral search coil in a magnetic Field. IEEE Trans Biomed Eng 10:137-145. Medline

Schiller PH, Tehovnik EJ (2001) Look and see: how the brain moves your eyes about. Prog Brain Res 134:127-142. CrossRef Medline

Schiller PH, Haushofer J, Kendall G (2004a) How do target predictability and precueing affect the production of express saccades in monkeys? Eur J Neurosci 19:1963-1968. CrossRef Medline

Schiller PH, Haushofer J, Kendall G (2004b) An examination of the variables that affect express saccade generation. Vis Neurosci 21:119-127. CrossRef Medline

Shadlen MN, Newsome WT (2001) Neural basis of a perceptual decision in the parietal cortex (area LIP) of the rhesus monkey. J Neurophysiol 86: 1916-1936. Medline

Snyder LH, Batista AP, Andersen RA (1997) Coding of intention in the posterior parietal cortex. Nature 386:167-170. CrossRef Medline
Sokolov EN (1963) Higher nervous functions; the orienting reflex. Annu Rev Physiol 25:545-580. CrossRef Medline

Sommer MA, Wurtz RH (2000) Composition and topographic organization of signals sent from the frontal eye field to the superior colliculus. J Neurophysiol 83:1979-2001. Medline

Sparks D, Rohrer WH, Zhang Y (2000) The role of the superior colliculus in saccade initiation: a study of express saccades and the gap effect. Vision Res 40:2763-2777. CrossRef Medline

Thier P, Andersen RA (1998) Electrical microstimulation distinguishes distinct saccade-related areas in the posterior parietal cortex. J Neurophysiol 80:1713-1735. Medline

Waitzman DM, Ma TP, Optican LM, Wurtz RH (1991) Superior colliculus neurons mediate the dynamic characteristics of saccades. J Neurophysiol 66:1716-1737. Medline

Zhang M, Barash S (2000) Neuronal switching of sensorimotor transformations for antisaccades. Nature 408:971-975. CrossRef Medline

Zhang M, Barash S (2004) Persistent LIP activity in memory antisaccades: working memory for a sensorimotor transformation. J Neurophysiol 91: 1424-1441. Medline 